\title{
Emodin enhances ATRA-induced differentiation and induces apoptosis in acute myeloid leukemia cells
}

\author{
YINGYU CHEN ${ }^{*}$, JING LI* , JIANDA HU, JING ZHENG, ZHIHONG ZHENG, \\ TINGBO LIU, ZHENXING LIN and MINHUI LIN \\ Fujian Medical University Union Hospital, Fujian Institute of Hematology, \\ Fujian Provincial Key Laboratory of Hematology, Fuzhou 350001, Fujian, P.R. China
}

Received June 2, 2014; Accepted July 21, 2014

DOI: $10.3892 /$ ijo.2014.2610

\begin{abstract}
Emodin, an extracted natural compound from the root and rhizome of Rheum palmatum L, has been shown to have multiple biological activities including anticancer functions in previous studies. In this study, we investigated the anti-leukemic activity of emodin alone or emodin in the presence all-trans retinoic acid (ATRA) in acute myeloid leukemia (AML) cells and the potential signaling pathway involved. We demonstrated that emodin could significantly enhance the sensitivity to ATRA and present additive differentiation-inducing effects in AML cell line NB4 cells and, especially, in NB4-derived ATRA-resistant MR2 cells. Further study showed that increasing dose of emodin could effectively induce growth inhibition and apoptotic effects in both cell lines as well as in primary leukemic cells from AML patients. Moreover, the apoptotic induction in AML cells was associated with the activation of caspase cascades involving caspase-9, caspase-3, and poly(ADP-ribose) polymerase (PARP) cleavage. In addition, leukemic cell response to emodin stimuli in vitro was observed through the decreased expression levels of $\mathrm{Bcl}-2$ and retinoic acid receptor $\alpha(\mathrm{RAR} \alpha)$. Importantly, emodin was demonstrated as a new inhibitor of PI3K/Akt in AML cells, even in primary AML cells. It inhibited Akt phosphoration (p-Akt) at Ser473 as efficiently as mTOR at Ser2448. Consistently, it exerted suppression effects on the phosphoration of mTOR downstream targets, 4E-BP1 and p70S6K. Taken together, these findings indicate that emodin might be developed as a promising anti-leukemic agent to improve the patient outcome in AML.
\end{abstract}

Correspondence to: Dr Jianda Hu, Fujian Institute of Hematology, Fujian Medical University Union Hospital, 29 Xinquan Road, Fuzhou, Fujian 350001, P.R. China

E-mail: jdhu@medmail.com.cn

*Contributed equally

Key words: emodin, apoptosis, differentiation, PI3K/Akt signaling pathway, acute myeloid leukemia

\section{Introduction}

Acute myeloid leukemia (AML) is characterized by differentiation arrest and inappropriate proliferation and survival of immature myeloid progenitors. In the majority of patients with AML who achieve a complete remission (CR), the leukemia will recur within 3 years after diagnosis. The survival probability at 1 and 5 year is 70 and 46\%, respectively, based on the favorable risk evaluation (1). Acute promyelocytic leukemia (APL) is a unique subtype of AML. APL patients achieve CR with all-trans retinoic acid (ATRA) treatment. However, the remission duration is short due to rapid development of retinoid resistance in some APL patients (2). Deregulation of phosphatidylinositol 3-kinase (PI3K)/Akt pathway may contribute to tumorigenesis, metastasis, and resistance to conventional chemotherapy. PI3K/Akt signaling pathway is frequently activated in AML cells, and it has been considered as a new target of therapeutic intervention for AML patients (3-7). Thus, the interruption of PI3K/Akt signaling pathway should be considered when designing anti-AML therapeutic strategies.

Emodin is a natural component extracted from the root and rhizome of Rheum palmatum L. It has multiple biological activities including antimicrobial, antiviral, anti-inflammatory, anti-ulcerogenic, immunosuppressive and chemo-preventive activities (8-10). The investigations of emodin-mediated anticancer effects are in progress. For example, emodin, as a tyrosine kinase inhibitor, suppressed growth of HER-2/neu overexpressing breast cancer cells in vivo (11). Emodin and docosahexaenoic acid (DHA) potently promoted arsenic trioxide $\left(\mathrm{As}_{2} \mathrm{O}_{3}\right)$ and interferon- $\alpha$ (IFN- $\alpha$ )-induced cell death in HTLV-I infected cells by generation of reactive oxygen species and inhibition of Akt and AP-1 pathways (12). It was also displayed that emodin enhanced the activity of gemcitabine against pancreatic cancer in mice by promoting the mitochondrial-dependent apoptotic pathway (13). Previous studies in our group have shown that emodin may induce apoptosis and reverse multidrug resistance in HL-60 and HL-60/ADR cells (14-16), but the molecular mechanisms have not been completely elucidated. In the present study, we provided the first demonstration of the potential roles and mechanisms of emodin alone or in combination with ATRA in NB4 cells, ATRA-resistant MR2 cells and especially those 
primary leukemic cells from newly diagnosed AML patients. We showed that low dose of emodin could potentially enhance ATRA-induced terminal differentiation in AML cells, especially, in ATRA-resistant cells. Emodin could effectively inhibit proliferation and induce apoptosis with a dose- and time-dependent manner in AML cells as well as ATRAresistant leukemic cells. The critical mechanism underlying these effects is linked to the inhibition of PI3K/Akt signaling pathway in AML cells. These results suggest that emodin or emodin in combination with ATRA may have benefits in AML therapy.

\section{Materials and methods}

Chemicals and reagents. Emodin $\left(\mathrm{C}_{15} \mathrm{H}_{10} \mathrm{O}_{5}, \mathrm{MW}: 446.35\right.$, HPLC-determined purity $>98 \%$ ) was obtained from Nanjing Qingze Medical Technology Co. (Nanjing, China). Emodin and ATRA (Sigma, St. Louis, MO, USA) were reconstituted in $100 \%$ dimethyl sulfoxide (DMSO) to $50,000 \mu \mathrm{M}$ as a stock solution. The stock solutions were maintained at $-20^{\circ} \mathrm{C}$ and further diluted in culture media before use.

Patients and cell lines. ATRA-resistant MR2 cell line and its parental NB4 cell line were obtained from Shanghai Institute of Hematology, Shanghai Ruijing Hospital, China. Cell lines were cultured in RPMI-1640 supplemented with 10\% FBS, penicillin $(100 \mathrm{IU} / \mathrm{ml})$ and streptomycin $(100 \mu \mathrm{g} / \mathrm{ml})$ based on the supplier's instructions and established procedures. The primary leukemia cells from peripheral blood samples were obtained from 21 newly diagnosed AML patients. Samples had $\geq 70 \%$ blasts in the initial isolation prior to manipulation. Normal controls were recruited from 6 healthy donors. All patient samples were referred to our hospital for cytomorphological and cytogenetic diagnostics, and were diagnosed as AML according to standard French-American-British (FAB) and WHO criteria. Peripheral blood mononuclear cells (PBMCs) were isolated by Ficoll-Paque density-gradient separation. Informed consent was obtained from all patients and healthy donors in accordance with the Declaration of Helsinki, and all manipulations of human specimens were approved by the institutional review board in Fujian Medical University Union Hospital.

Cell proliferation assay. NB4, MR2 and primary AML cells plated in 96-well plates were treated in triplicates with emodin, ATRA alone or emodin plus ATRA for 48 or $72 \mathrm{~h}$ at $37^{\circ} \mathrm{C}$. Cells were then incubated with $5 \mathrm{mg} / \mathrm{ml}$ MTT (Sigma) for $4 \mathrm{~h}$. The supernatants were removed and cells were pulsed with $100 \mu 1$ DMSO. The optical density (OD) was measured at 492/630 nm using a spectrophotometer (STAT FAX-2100). The inhibitory rate on cell proliferation was calculated as $\left(1-\mathrm{OD}_{\text {treated }} / \mathrm{OD}_{\text {control }}\right) \times 100 \%$. The half inhibitory concentration $\left(\mathrm{IC}_{50}\right)$ values were obtained by the Logit method.

Cell differentiation assay. NB4 and MR2 cells were seeded at a density of $5.0 \times 10^{4} / \mathrm{ml}$ in 6 -well plates in the presence of either $10 \mu \mathrm{M}$ emodin, $1.0 \mu \mathrm{M}$ ATRA alone or $10 \mu \mathrm{M}$ emodin in combination with $1.0 \mu \mathrm{M}$ ATRA at $37^{\circ} \mathrm{C}$ for $96 \mathrm{~h}$. Cells were harvested, washed twice with phosphate-buffered saline (PBS). Cell differentiation effects were determined by morphological examination, CD11b analysis and NBT staining. Cell morphological features were observed by microscopy after Wright-Giemsa staining. Cell surface differentiation antigen CD11b (Biolegend, San Diego, CA, USA) was measured according to the manufacture's instruction. Data were acquired by flow cytometry (Beckman Coulter FC500, Fullerton, CA, USA). The nitroblue tetrazolium chloride (NBT) reduction assay was performed as previously described $(17,18)$. Total of 200 cells on each slide were counted under light microscope, and the percentages of NBT-positive cells were calculated in each group.

Apoptosis assay. Briefly, 1.0x10 $/ \mathrm{ml}$ of NB4 and MR2 cells in RPMI-1640 medium with 10\% fetal bovine serum were plated in 6-well plates. After $12 \mathrm{~h}$ of incubation with emodin, cells were harvested and washed with PBS, and then stained with Annexin V-FITC/PI (Becton-Dickinson, NJ, USA) according to the manufacturer's instructions. The early apoptotic cells were quantified by flow cytometry. To further analyze the cell cycle distribution and the percentages of late apoptotic cells, NB4 and MR2 cells were harvested after $48 \mathrm{~h}$ of treatment with emodin. Cell pellets were incubated with the DNA PREP LPR solutions (Beckman Coulter), followed by staining with propidium iodide on ice. Flow cytometry analysis was performed to determine the fraction of cells in $G_{0} / G_{1}, S$, and $\mathrm{G}_{2} / \mathrm{M}$. The cells undergoing apoptosis were obtained from the distinct sub- $\mathrm{G}_{1}$ region of the DNA distribution histograms.

DNA fragmentation assay. NB4, MR2 and primary AML cells were exposed to different concentrations of emodin for $48 \mathrm{~h}$. Cells were harvested by centrifugation. After washed with PBS, DNA fragmentation was analyzed using the manufacturer's procedures (Beyotime, Shanghai, China). In brief, genomic DNA was extracted, then absorbed by a miniprep spin column and eluted with buffer. Electrophoresis for purified DNA was performed in a $1.5 \%$ agarose gel at $35 \mathrm{~V}$ for $3 \mathrm{~h}$. DNA was visualized by Goldview Nucleic Acid Stain on Gel Image Analysis System (Peiqing, JS-380A, Shanghai, China).

Western blotting. NB4, MR2 and primary AML cells were exposed to emodin at varying concentrations and time points. Total protein was extracted and western blotting was performed as described previously (18). Quantification of the band densitometry was performed by Quantity One Version 4.6.2 Software (Bio-Rad, Hercules, CA, USA). Primary antibodies against human caspase-9, caspase-3, poly(ADP-ribose) polymerase (PARP), p-Akt (Ser473), Akt, p-mTOR (Ser2448), mTOR, 4E-BP1 (53H11), p-4E-BP1 (Thr70), p70S6K and p-p70S6K (Thr389) were obtained from Cell Signaling Technology (Beverly, MA, USA). Bcl-2, RAR $\alpha$ and $\beta$-actin antibodies were purchased from Santa Cruz Biotechnology, Inc. (Santa Cruz, CA, USA). Rapamycin was obtained from Calbiochem EMD Millipore Corp. (Billerica, MA, USA). LY294002 and PD98059 were obtained from Sigma-Aldrich Corp. (St. Louis, MO, USA).

Statistical analysis. Results are presented as mean \pm standard deviation, and statistical comparisons of experimental groups were evaluated by Student's t-test. Statistical significance was defined as $\mathrm{P}<0.05$. 

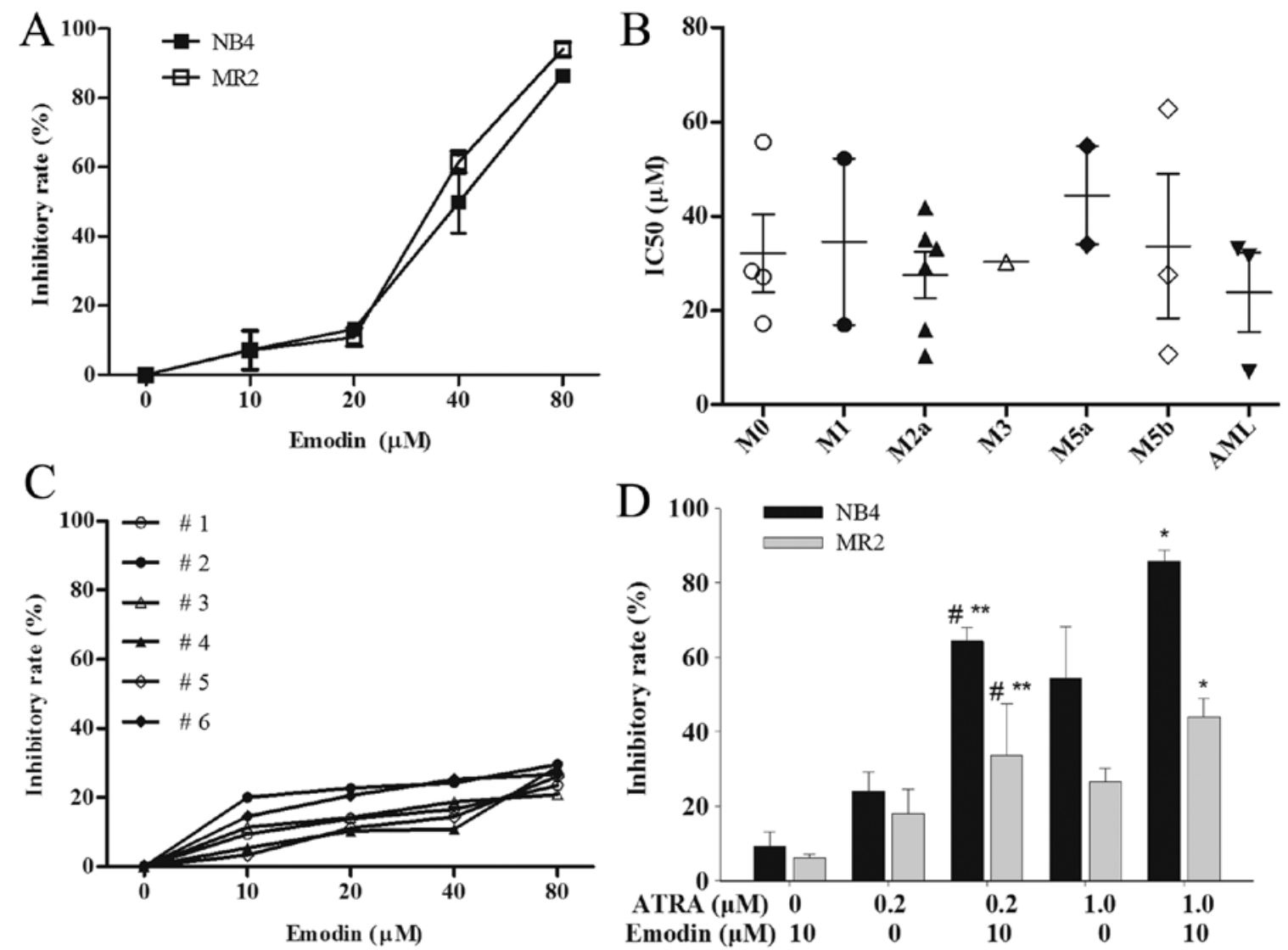

Figure 1. Effects of emodin, or emodin in combination with ATRA on the proliferation of APL cell lines, primary AML cells and normal PBMCs. (A) Dosedependent inhibition of proliferation in NB4 cells and NB4-derived ATRA-resistant MR2 cells treated with the indicated concentrations of emodin for $48 \mathrm{~h}$, as demonstrated by MTT assay. (B) Primary AML cells were treated with increasing doses of emodin (10-80 $\mu \mathrm{M})$ for $72 \mathrm{~h}$. IC $\mathrm{C}_{50}$ values of 21 AML patient samples with different FAB classification subtypes were calculated. (C) Freshly isolated peripheral blood mononuclear cells (PBMCs) from 6 healthy donors were cultured with the indicated concentrations of emodin for $72 \mathrm{~h}$. (D) NB4 and MR2 cells were incubated with emodin (10 $\mu \mathrm{M})$, ATRA $(0.2$ and $1.0 \mu \mathrm{M})$ alone, or their combination for $48 \mathrm{~h}$. The value presented as mean $\pm \mathrm{SD}$ from three independent experiments. ${ }^{*} \mathrm{P}<0.05$, versus $0.2 \mu \mathrm{M}$ ATRA group; ${ }^{* *} \mathrm{P}>0.05$, versus $1.0 \mu \mathrm{M}$ ATRA group; ${ }^{*} \mathrm{P}<0.05$, versus $1.0 \mu \mathrm{M}$ ATRA group.

\section{Results}

Emodin inhibits cell proliferation in NB4 cells, MR2 cells and primary AML cells. MTT assay was used to examine the effects of emodin on ATRA-resistant MR2 cells and the parental NB4 cells. Consistent with the results of our previous study in HL-60 and HL-60/ADR cells, NB4 and MR2 cells were also sensitive to emodin in a dose-dependent manner (Fig. 1A). Emodin inhibited MR2 cell proliferation with an average $\mathrm{IC}_{50}$ value of $34.01 \pm 2.40 \mu \mathrm{M}$, which was similar to the effect on the parental NB4 cells yielding an average $\mathrm{IC}_{50}$ of $37.99 \pm 2.30 \mu \mathrm{M}$. To identify whether similar effects were found in the primary AML cells, 21 samples from AML patients were treated with increasing concentrations of emodin (ranging from 10 to $80 \mu \mathrm{M}$ ) in vitro for $72 \mathrm{~h}$. As shown in Fig. 1B, the results demonstrated that all of the primary AML cells isolated from 21 different subtypes of AML patients including three AML cases unclassified, although variable in degree, were sensitive to emodin with the average $\mathrm{IC}_{50}$ value of $31.22 \pm 15.58 \mu \mathrm{M}$. While less cytotoxicity was found when healthy PBMCs were exposed to the same setting of emodin in vitro. More than $70 \%$ cells from the 6 healthy donors remained alive even though administered with emodin as high as $80 \mu \mathrm{M}$ (Fig. 1C).
Enhancements of the sensitivity of ATRA in NB4 and MR2 cells were also observed by MTT assay. ATRA at $0.2 \mu \mathrm{M}$ and emodin at $10 \mu \mathrm{M}$ alone only induced mild proliferation inhibition in NB4 and MR2 cells. However, the combination of $0.2 \mu \mathrm{M}$ ATRA and $10 \mu \mathrm{M}$ emodin significantly raised inhibitory rates in NB4 cells and MR2 cells $(\mathrm{P}<0.05)$, which were similar to those achieved by $1 \mu \mathrm{M}$ ATRA treatment in the two cell lines $(\mathrm{P}>0.05)$. When increased up the concentration of ATRA to $1 \mu \mathrm{M}$ in combination with $10 \mu \mathrm{M}$ of emodin, the average levels of growth inhibition were significantly enhanced from $54.29 \pm 13.85$ and $26.58 \pm 3.57 \%$ in $1 \mu \mathrm{M}$ ATRA mono-treatment group to $85.77 \pm 2.89$ and $43.95 \pm 4.99 \%$ in the combination group in NB4 and MR2 cells, respectively, $(\mathrm{P}<0.05)($ Fig. 1D).

Emodin enhances differentiation induction of ATRA in retinoid-responsive NB4 cells as well as in retinoid-resistant MR2 cells. As shown in Fig. 1D, >90\% NB4 and MR2 cells remained alive when treated with emodin at a concentration of $10 \mu \mathrm{M}$. To identify whether this non-toxic dose of emodin may enhance ATRA-sensitivity in NB4 cells, especially in its ATRA-resistant subclone MR2 cells, we detected the cellular differentiation effects of $10 \mu \mathrm{M}$ emodin combined with $1.0 \mu \mathrm{M}$ ATRA in the two cell lines. Compared with the 

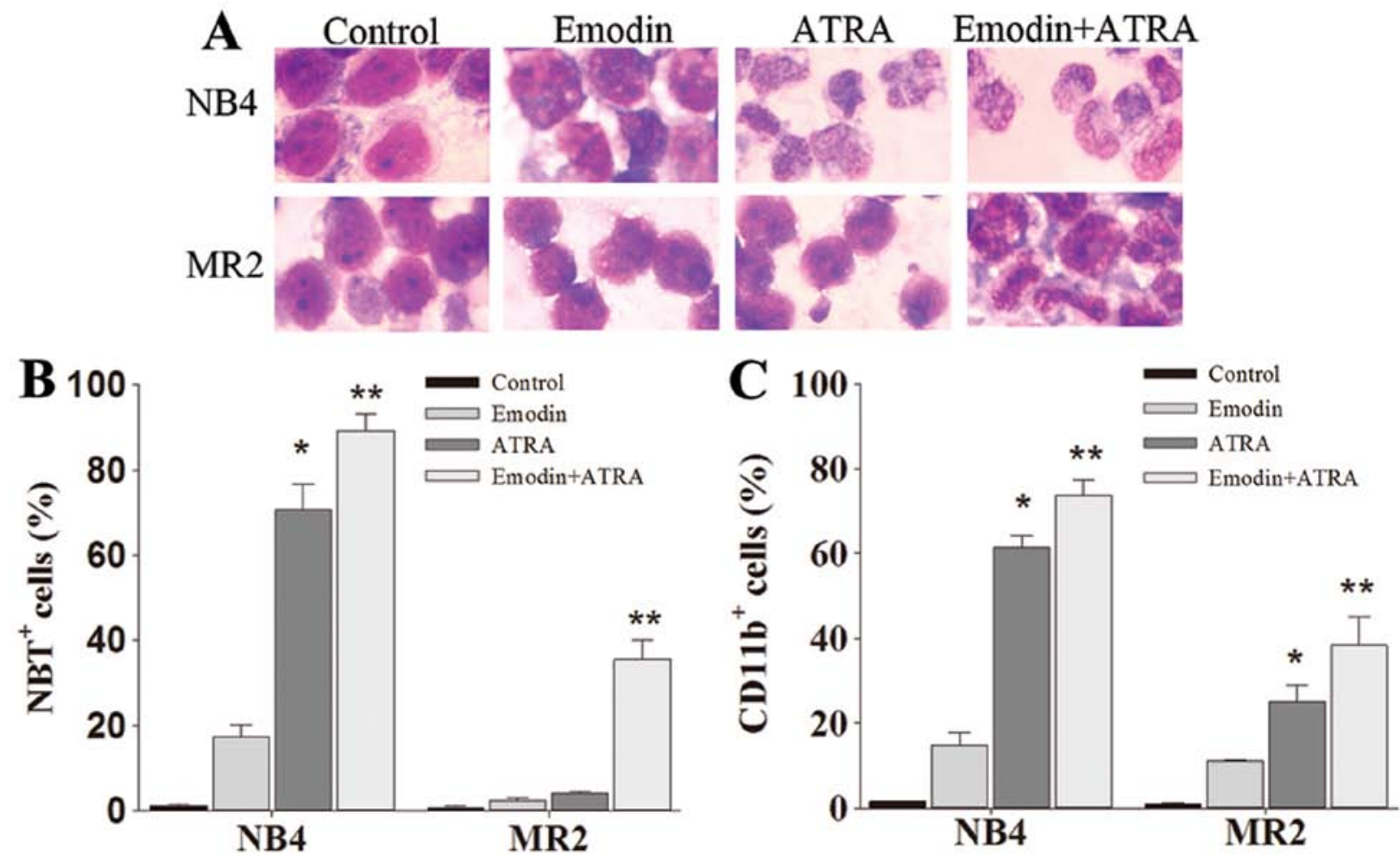

Figure 2. Emodin enhances the differentiation induction effects of ATRA in NB4 cells and MR2 cells. NB4 and MR2 cells were incubated with $10 \mu$ M emodin in the presence or absence of $1.0 \mu \mathrm{M}$ ATRA for $96 \mathrm{~h}$. (A) Harvested cells were stained by Wright-Giemsa method. (B) NBT-positive cells were analyzed by flow cytometry. (C) CD11b-positive cell percentages were calculated. The experiments were repeated three times. ${ }^{*} \mathrm{P}<0.01$, versus control group; ${ }^{* *} \mathrm{P}<0.05$, versus either emodin or ATRA group.

untreated and ATRA-treated cells, emodin only-treated cells presented slight differentiation-inducing effects in either NB4 cells or MR2 cells. As shown in Fig. 2A, the combination of emodin and ATRA synergistically induced terminal granulocytic differentiation manifested as smaller cell size, the disappearance of nucleoli, reduced nuclei-cytoplasm ratio, and condensed, distorted and stab form nuclei. Moreover, the acceleration processes of NB4 and MR2 cell differentiation after the combination treatment were further confirmed by CD11b expression analysis and NBT reduction assay. The averaged levels of CD11b-positive cells in co-treatment group were $73.67 \pm 3.76 \%$ in NB4 cells and $38.29 \pm 6.68 \%$ in MR2 cells, NBT-positive cells were $89.17 \pm 4.01$ and $35.50 \pm 4.44 \%$, respectively. CD11b and NBT-positive cells in the combination treatment group were significantly higher than those obtained from either emodin or ATRA alone group $(\mathrm{P}<0.05)$ (Fig. 2B and $\mathrm{C}$ ).

Emodin induces cell apoptosis in NB4 cells, MR2 cells and primary AML cells. Next, we assessed the role of emodin on the induction of apoptosis in AML cells. The results showed that emodin exerted apoptotic effects in NB4 and MR2 cells dose-dependently, as assessed by Annexin V-FITC/PI staining assay (Fig. 3A). Quantitative evaluation of apoptotic cells was further determined using cell cycle analysis. When compared with the control group, the sub- $\mathrm{G}_{1}$ (apoptotic cell) population was clearly observable in NB4 and MR2 cells treated with $30 \mu \mathrm{M}$ emodin. The ratio of apoptotic cells was $21.37 \pm 0.72$ and $26.78 \pm 5.19 \%$ in NB4 and MR2 cells, respectively (Fig. 3B). Results also revealed a marked $G_{0} / G_{1}$ and $G_{2} / M$ phase arrest in
NB4 and MR2 cells in the presence of emodin. Furthermore, we observed significantly decreased $\mathrm{S}$ phase cell population in the two cell lines under the same experimental condition (Fig. 3C and D). DNA fragmentation assay was applied to assess the effect of emodin on the induction of apoptosis in AML cells. Consistently, we found that both of NB4 and MR2 cells succumbed to emodin-induced the formation of DNA fragments at 48-h treatment (Fig. 3E). Moreover, primary AML cells were also sensitive to emodin stimuli in vitro. As shown in Fig. 3F, the apoptotic DNA fragmentation was present in each emodin-treated primary AML sample group, which further confirmed that emodin exhibited apoptotic induction effects in AML cells.

Emodin leads to activation of the caspase-dependent pathway and the degradation of RAR $\alpha$ protein in NB4 and MR2 cells. The mechanisms underlying emodin-induced apoptosis in AML cells were also analyzed. As shown in Fig. 4A, emodin treatment reduced the expression levels of procaspase- 3 and procaspase-9 in NB4 and MR2 cells in a concentration- and time-dependent manner. The cleavage of caspase-3 was induced in both cell lines. Consistently, results also showed that emodin induced cleavage of PARP, one of the important caspase-3 substrates. PARP was activated by cleaved 116-kDa fragment to $85-\mathrm{kDa}$ fragment. The activation modulated by emodin was enhanced with the longer incubation time and higher dose exposure.

Anti-apoptotic protein, Bcl-2, plays an important role during caspase cascade activation and apoptosis induction. PML/RAR $\alpha$ oncoprotein was specifically expressed in APL 

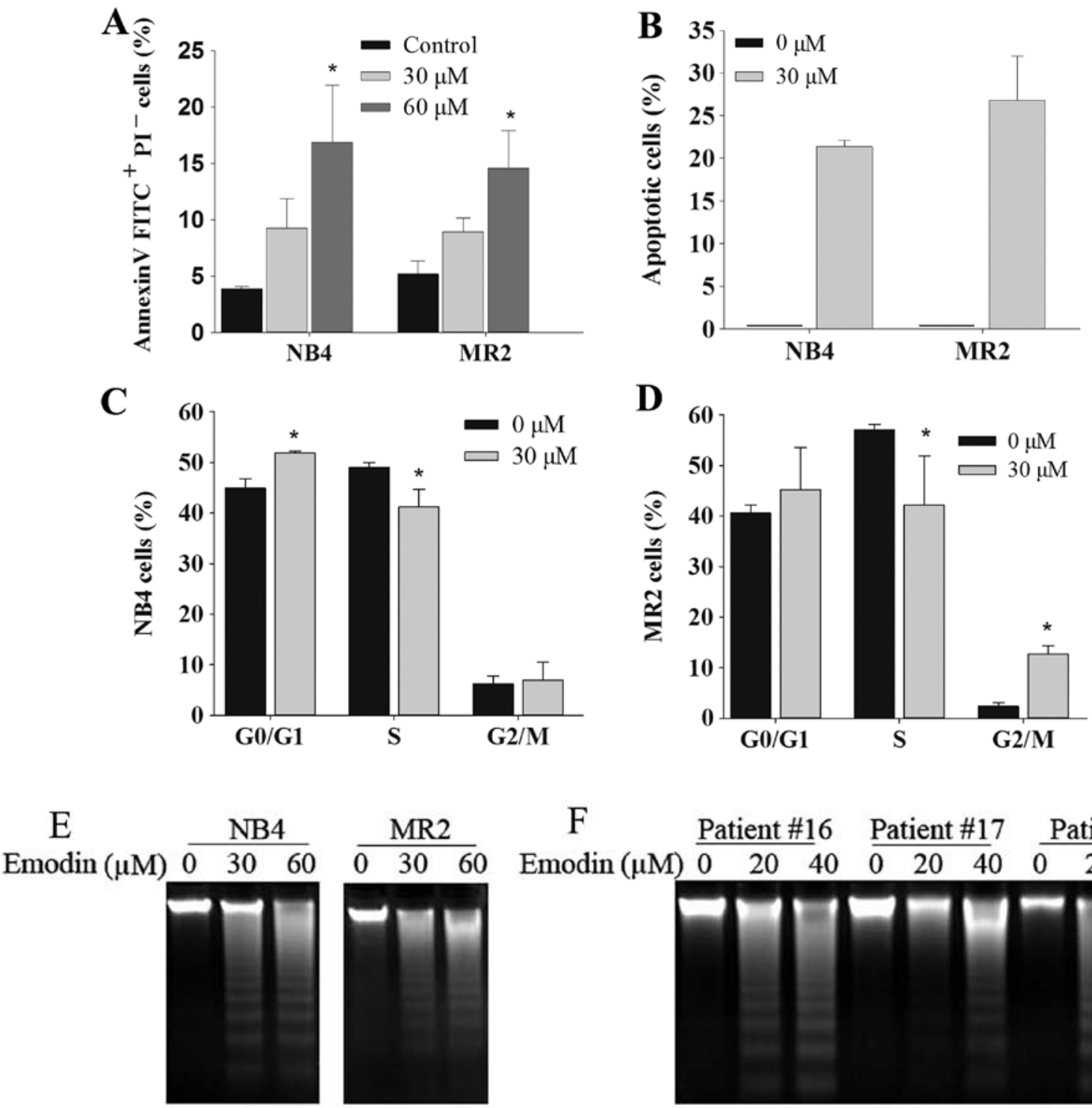

$\mathrm{F}$

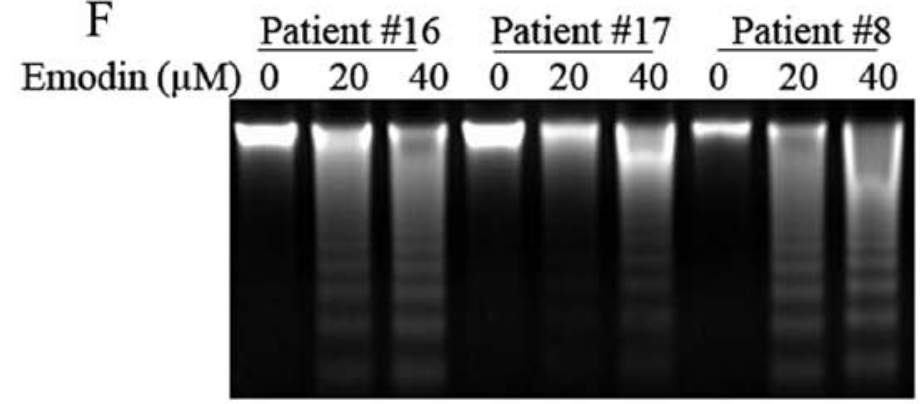

Figure 3. Emodin induces apoptosis in NB4, MR2 and primary AML cells. (A) Analysis of apoptotic cells by Annexin V-FITC/PI staining. NB4 and MR2 cells were treated with 30 and $60 \mu \mathrm{M}$ emodin for $12 \mathrm{~h}$. The data presented as mean $\pm \mathrm{SD}$ from three independent experiments. * $\mathrm{P}<0.01$, versus control group. (B-D) Emodin induced cell cycle arrest in NB4 and MR2 cells. NB4 and MR2 cells were cultured in the presence or absence of $30 \mu \mathrm{M}$ emodin for $48 \mathrm{~h}$, and cell cycle analysis was performed by PI staining. Apoptotic cell percentages were obtained from sub- $\mathrm{G}_{1}$ cell population. All values represent mean \pm SD of three independent experiments. ${ }^{*} \mathrm{P}<0.05$, versus control group. (E and F) NB4, MR2 cells and primary AML cell samples were treated with indicated concentrations of emodin for $48 \mathrm{~h}$. Genomic DNA was purified and subjected to electrophoresis in $1 \%$ agarose gel. The assay was repeated at least twice. Representative results from 3 out of 21 primary AML samples are shown (F).

cells, which inhibits the granulocyte development at the promyelocytic stage of differentiation. Therefore, we further examined the changes of expression levels of Bcl-2 and RAR $\alpha$ protein in NB4 and MR2 cells following the treatment with emodin. Spot densitometry quantification analysis revealed a decrease in Bcl-2 expression from 52 to $26 \%$ in NB4 cells and from 82 to $22 \%$ in MR2 cells, respectively, when administered with increasing doses of emodin at different time points. Although RAR $\alpha$ protein was not affected in NB4 cells with the addition of $30 \mu \mathrm{M}$ emodin for $15 \mathrm{~h}$, decreased RAR $\alpha$ ranging from 49 to $37 \%$ were observed when longer incubation time and higher dose were applied. Similarly, the presence of emodin also resulted in a dose- and time-dependent degradation (ranging from 69 to $14 \%$ ) of RAR $\alpha$ protein in MR2 cells (Fig. 4A).
Emodin inhibits activation of the PI3K/Akt signaling pathway in AML cells. Constitutive PI3K/Akt activation is frequently observed in AML samples and sustains leukemic cell growth (5-7,19,20). Hence, we tested whether emodin may affect PI3K/Akt signaling pathway in AML cells. The results demonstrated that emodin markedly abrogated phosphorylation of Akt at Ser473 and of mTOR at Ser2448 in both NB4 and MR2 cells. Of note, emodin also inhibited mTOR downstream targeted proteins p-4E-BP1 and p-p70S6K in the two cell lines in a dose- and time-dependent manner (Fig. 4A). To further validate our observation that emodin downregulates the activation of crucial molecules in PI3K/Akt pathway, PI3K/Akt inhibitor (LY294002), mTOR inhibitor (rapamycin) and MAPK inhibitor (PD 98059) were administered parallelly with two different concentrations of emodin in NB4 and 
A

NB4

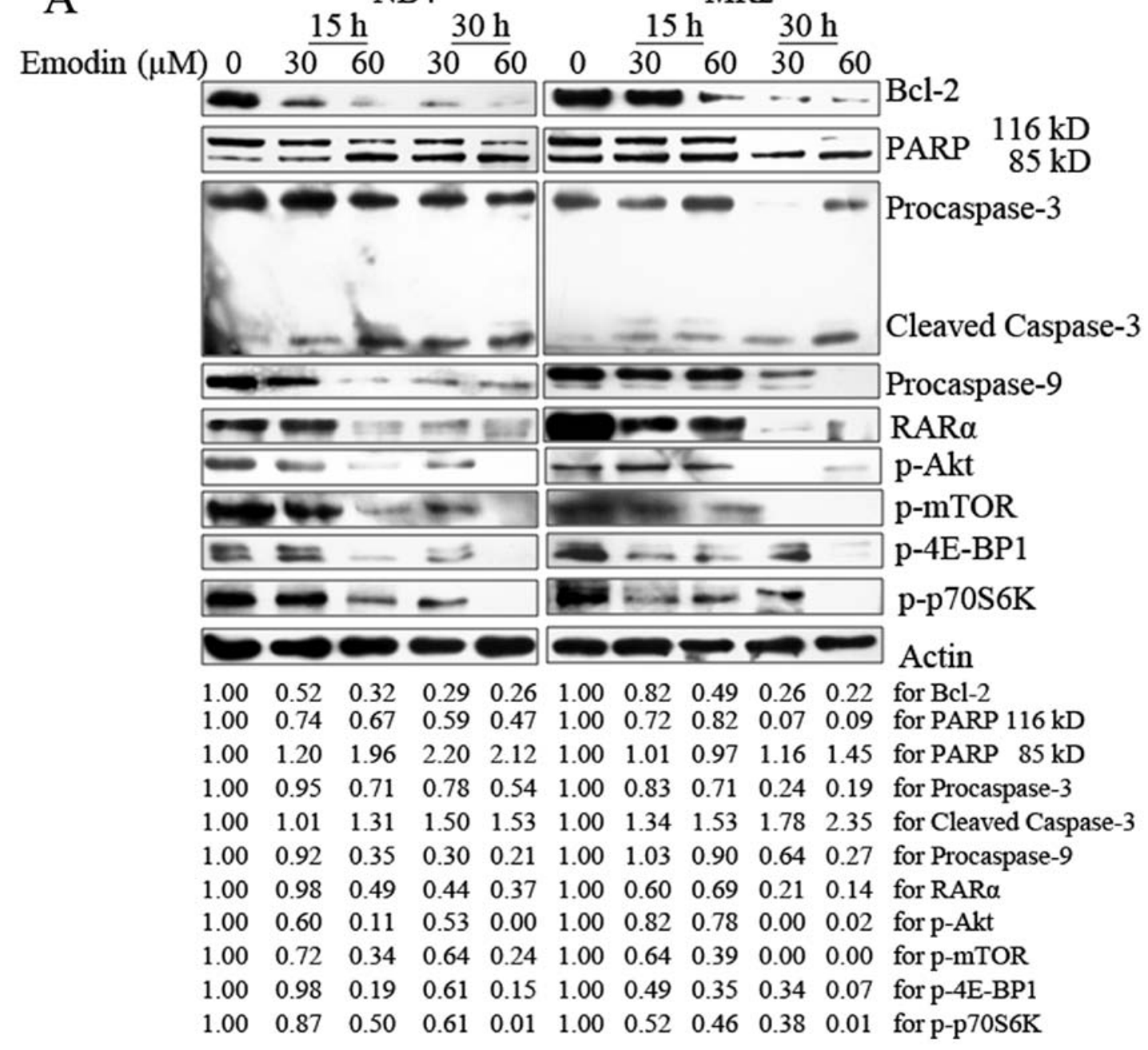

B
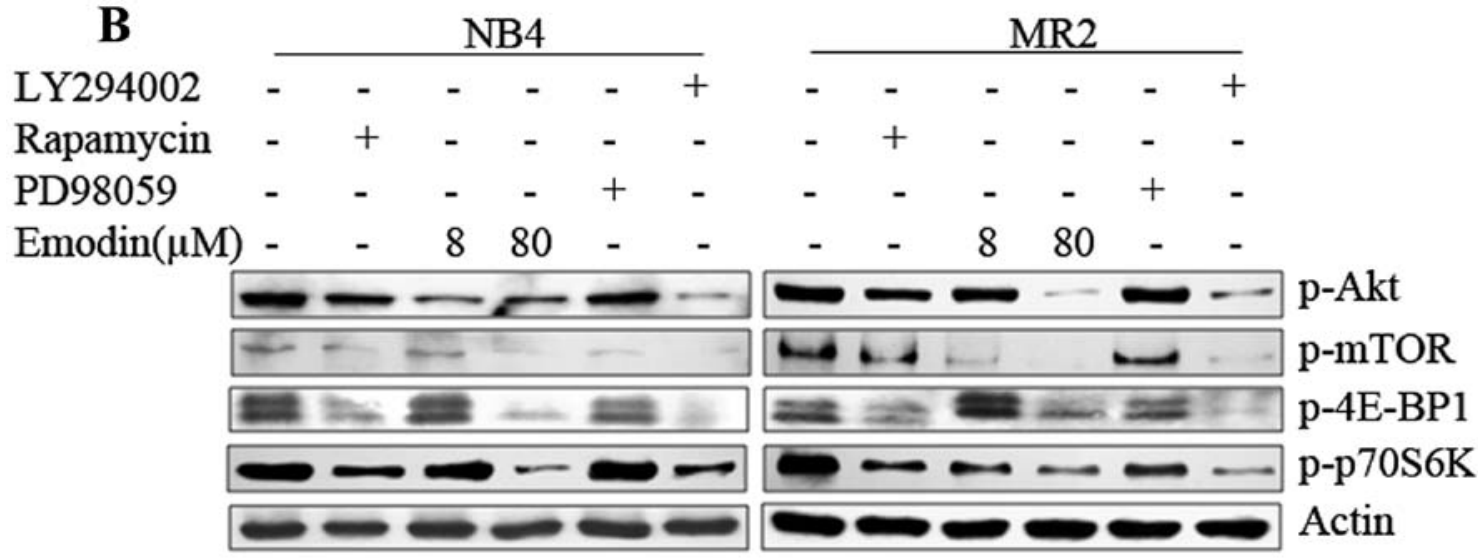

Figure 4. Emodin activates the caspase-dependent apoptotic pathway and inhibits PI3K/Akt signaling pathway in NB4 and MR2 cells. (A) NB4 and MR2 cells were treated with emodin at indicated concentrations and time-points. Proteins were extracted from harvested cells. Western blotting was performed to visualize the expression of Bcl-2, $116 \mathrm{kDa}$ PARP, $85 \mathrm{kDa}$ PARP, procaspase-3, cleaved caspase-3, procaspase-9, RAR $\alpha$, phosphorylation of Akt (Ser473), mTOR (Ser2448), 4E-BP1 (Thr70) and p-70S6K (Thr389) proteins along with $\beta$-actin as loading controls. The experiments were repeated three times. The intensity of different bands was quantified. Changes in protein expression levels are represented as percentage change from untreated control levels, which were set to $100 \%$ (1.00). The lower set of numbers indicates the relative protein level. (B) NB4 and MR2 cells were cultured with emodin (8 and $80 \mu \mathrm{M})$, LY294002 $(40 \mu \mathrm{M})$, rapamycin $(1.0 \mu \mathrm{M})$ and PD98059 $(20 \mu \mathrm{M})$ for $12 \mathrm{~h}$. Protein extracts from harvested cells were subjected to western blotting using anti-p-Akt, antip-mTOR, anti-p-4E-BP1 and anti-p-p70S6K antibodies. All experiments were repeated three times.

MR2 cells. Compared with the untreated group and the lower dose of emodin $(8 \mu \mathrm{M})$ treatment group, the inhibitory effects on the phosporylation of Akt, mTOR, 4E-BP1 and p70S6K induced by $80 \mu \mathrm{M}$ emodin were as strong as those following by LY294002 and rapamycin treatment. As expected, neither Akt nor mTOR or its downstream target activity in PI3K/Akt pathway were suppressed by PD 98059 in NB4 and MR2 cells (Fig. 4B). 


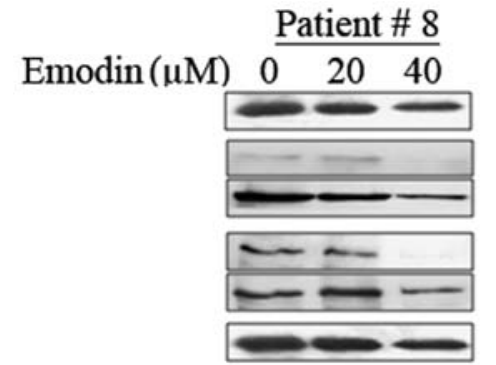

$\begin{array}{lll}1.00 & 0.87 & 0.78\end{array}$

$\begin{array}{lll}1.00 & 0.82 & 0.69\end{array}$

$\begin{array}{lll}1.00 & 0.69 & 0.12\end{array}$

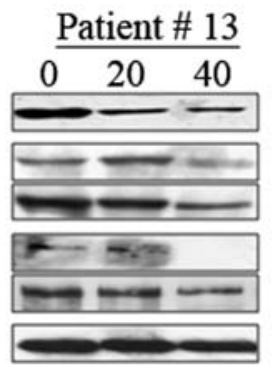

$\begin{array}{lll}1.00 & 0.52 & 0.41\end{array}$

$\begin{array}{lll}1.00 & 1.00 & 0.72\end{array}$

$\begin{array}{lll}1.00 & 0.99 & 0.13\end{array}$

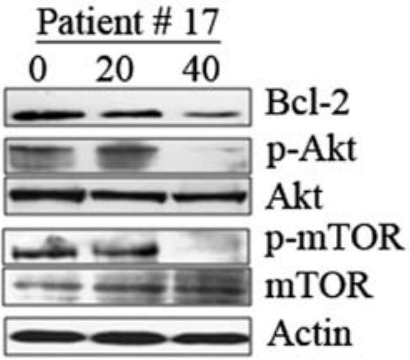

$\begin{array}{lll}1.00 & 0.63 \quad 0.39 \text { for Bcl-2 }\end{array}$

$\begin{array}{lll}1.00 & 0.66 & 0.20 \text { for } \mathrm{p}-\mathrm{Akt}\end{array}$ $1.00 \quad 0.860 .30$ for $\mathrm{p}$-mTOR

Figure 5. Emodin downregulates Bcl-2 expression and inhibits PI3K/Akt signaling pathway in primary AML cells. Primary AML cells were treated with indicated concentrations of emodin for $24 \mathrm{~h}$. Cells were harvested, and cell lysates were subjected to western blotting using anti-p-Akt (Ser473), anti-Akt, anti-p-mTOR (Ser2448), anti-mTOR, anti-Bcl-2 and anti- $\beta$-actin antibodies. $\beta$-actin was used as loading control. Band intensities were quantified. Changes in protein expression levels are presented as percentage change from untreated control levels, which were set to $100 \%$ (1.00). Three representative sample results (patient nos. 8, 13 and 17) are shown. Relative intensity of Bcl-2, p-Akt and p-mTOR is depicted by the lower set of numbers.

To identify the potential clinical efficacy using emodin as a new PI3K/Akt inhibitor, 21 primary AML samples were included in this study. Eighteen out of the 21 AML samples presented constitutive Akt activation. It is similar to previously reported data that Akt phosphorylation at Ser473 can be detected in $50-80 \%$ of AML patients $(5,21)$. Bcl-2 protein could be seen in all of the 21 patient samples as well. The results show that emodin suppressed Akt phosphorylation in a dose-dependent manner among these 18 primary AML samples, which was consistent with the results we observed in NB4 and MR2 cells. Similar downregulation effects on Bcl-2 expression were found in all of the 21 detected samples. The phosphorylation form of mTOR was detectable in 8 out of the 18 AML samples with constitutive Akt activation. Interestingly, the presence of increasing doses of emodin significantly abrogated mTOR activation along with phosphorylated Akt inhibition in these 8 samples. The results from three representative AML samples (patient nos. 8, 13 and 17) are shown in Fig. 5. These strongly indicate that the inhibition of PI3K/Akt activation involved in the antileukemia activity is triggered by emodin.

\section{Discussion}

AML is a clonal hematopoietic stem cell disorder characterized by differentiation arrest, inappropriate proliferation and survival of immature myeloid progenitors. The prognosis of the disease remains poor, and the mortality rates in AML patients are high despite considerable improvements in therapy. The long-term follow-up studies showed a relapse rate exceeding $20 \%$ in high-risk APL patients (22-24), a unique subtype of AML, although the targeted therapy with ATRA and $\mathrm{As}_{2} \mathrm{O}_{3}$ was introduced in the clinic. The investigation of novel anti-leukemic agent might generate considerable benefits for AML patients.

The antitumor activities of emodin have been reported. For example, emodin in combination with clinically achievable doses of DHA reduced arsenic concentrations by 100 -fold while still remaining highly toxic to tumor cells (12). Emodin may improve the expression of globin genes in K562 cells and also induce K562 cells to erythroid differentiation possibly via upregulating ALAS2 and c-KIT and downregulating miR-221 and miR-222 (25). In this study, emodin exhibited significant anti-leukemic effects in vitro. We provided the first demonstration that nontoxic dose of emodin $\left(<\mathrm{IC}_{10}\right)$ inhibited growth and induced differentiation to a low degree in NB4 and MR2 cells. However, the administration of low concentration of emodin along with ATRA synergistically enhanced ATRA-induced terminal differentiation in NB4 cells. Especially, the emodin/ ATRA combination also significantly increased CD11bpositive cells, NBT-response and differentiation-related morphological features in ATRA-resistant MR2 cells. More interestingly, when increasing dose of emodin was administered individually, we found that it presented proliferation inhibition dose-dependently in the two cell lines as well as in primary leukemic cells from AML patients. We further showed that administration with emodin caused AML cells to undergo apoptosis as evidenced by increasing proportion of Annexin V-FITC-positive cells, sub- $\mathrm{G}_{1}$ cell cycle arrest and obvious DNA fragmentation.

The underlying mechanisms responsible for emodininduced apoptosis in AML cells were also explored in this study. Muto et al showed that the activation of caspase-3 and -9 can be triggered by emodin in multiple myeloma cells (26). AMAD, an emodin azide methyl anthraquinone derivative, was reported to induce apoptosis in the human breast cancer cell line MDA-MB-453 and the human lung adenocarcinoma Calu-3 cells via a collapse of the mitochondrial membrane potential and the activation of caspase cascades (27). As shown in this study, caspase-3, caspase-9 and PARP precursors were decreased, while the cleavage of caspase- 3 and PARP were induced in AML cells in response to emodin. Emodin triggered the activation of caspases in AML cells consistent with the previous findings in multiple myeloma cells, breast cancer cells and lung adenocarcinoma cells $(26,27)$.

Bcl-2 is one of the important anti-apoptotic proteins of the Bcl-2 family, which has been shown to contribute to drug resistance in various human malignancies including hematologic malignancies and solid tumors. Overexpression of 
$\mathrm{Bcl}-2$ is associated with poor prognosis (28-31). We observed that emodin suppressed Bcl-2 expression in ATRA-resistant MR2 cells as well as in its parental NB4 cells. Of note, dosedependent blockage of Bcl-2 expression was further identified in 21 cases of primary AML cell samples when cultured in the presence of emodin. An in vivo study in our group recently demonstrated that consecutive treatment with emodin could effectively downregulate $\mathrm{Bcl}-2$ in AML in a xenograft model in nude mice (data not shown). These indicate suppression of Bcl-2 may contribute apoptosis execution in emodin-treated AML cells.

The promyelocytic leukemia (PML)/RAR $\alpha$ fusion protein formed as a result of the $t(15 ; 17)$ translocation was found in most of APL patients. As retinoic acid receptors, APL blasts express large amounts of PML/RAR $\alpha$, RAR $\alpha$ and RXR isoforms. To activate PML/RAR $\alpha$ oncoprotein degradation appears to represent a critical parameter for successful treatment of APL (32-35). Broad networks of post-transcriptional suppressive pathways are activated during ATRA-induced growth inhibition processes in APL cells (36). Interestingly, we found emodin significantly induced loss of RAR $\alpha$ protein in the same manner as Bcl-2 protein in NB4, especially in MR2 cells. It remains to be further investigated how emodin exerts degradation effects on $\mathrm{RAR} \alpha$ protein.

The constitutive activation of Akt pathway plays a critical role in the progression of AML cases. In the present study, we assessed whether the inhibition of PI3K/Akt pathway was concurrently involved in emodin-mediated apoptosis in AML cells. PI3K/Akt signaling pathway is comprised of a family of intracellular protein kinases. Akt activation indirectly promotes transcription of anti-apoptotic genes, while it directly phosphorylates the downstream target, mTOR. Phosphorylated mTOR promotes cell cycle transition from the $\mathrm{G}_{1}$ to $\mathrm{S}$ phase via phosphorylation of the downstream targets p70S6K and 4E-BP1, which favor translation of mRNAs for certain growth-promoting proteins such as Cyclin D. We demonstrated that emodin is the direct inhibitor of Akt activation in AML cells. Importantly, it also inhibited mTOR leading to reduced phosphorylation of 4E-BP1 and p70S6K in AML cells. Accordingly, the downregulation of anti-apoptotic protein $\mathrm{Bcl}-2$, cell cycle arrest to $\mathrm{G}_{1}$ phase and reduction of $\mathrm{S}$ phase cell population were found in the emodin-treated leukemic cells. Of note, emodin abrogated phosphorylation of Akt at Ser473, which is the site responsible for activating Akt in a feedback loop and has been implicated in rapamycin failure after prolonged treatment $(6,7,37,38)$. Our results indicated emodin may prevent the feedback loop. Moreover, the inhibition of PI3K/Akt pathway by emodin was further confirmed by comparison with PI3K/Akt inhibitor LY294002 and mTOR inhibitor rapamycin, as well as MAPK inhibitor PD98059. Thus, the downregulation of PI3K/Akt pathway is an important molecular mechanism underlying emodininduced apoptosis in AML cells. Whether there are other pathways, such as ERK1/2 pathway, in response to emodin stimuli in AML cells will be further identified.

In conclusion, we have demonstrated that emodin could enhance ATRA-induced differentiation in APL cells, even in ATRA-resistant leukemic cells. We further showed that emodin induced AML cell apoptosis dose- and time-dependently through inhibition of the PI3K/Akt signaling pathway involving activation of the caspase cascades. To this end, emodin may be considered as a new promising anti-leukemic agent to overcome ATRA-resistance and to improve clinical outcome of AML. Therefore, it would be worthy of extensive experimental investigations in vivo.

\section{Acknowledgements}

This study was supported by National and Fujian Provincial Key Clinical Specialty Discipline Construction Program, China, National High Technology Research and Development Program of China, 863 program (2012AA02A505), National Public Health Grand Research Foundation (201202017), Fujian Provincial Key Laboratory Foundation of Hematology (2009J1004), Fujian Provincial Natural Science Foundation (2012J01353), Youth Foundation of Fujian Provincial Health Bureau (2010-2-15).

\section{References}

1. Dohner H, Estey EH, Amadori S, Appelbaum FR, Buchner T, Burnett AK, et al: Diagnosis and management of acute myeloid leukemia in adults: recommendations from an international expert panel, on behalf of the European LeukemiaNet. Blood 115: 453-474, 2010.

2. Zhou GB, Zhang J, Wang ZY, Chen SJ and Chen Z: Treatment of acute promyelocytic leukaemia with all-trans retinoic acid and arsenic trioxide: a paradigm of synergistic molecular targeting therapy. Philos Trans R Soc of Lond B Biol Sci 362: 959-971, 2007.

3. West KA, Castillo SS and Dennis PA: Activation of the PI3K/Akt pathway and chemotherapeutic resistance. Drug Resist Updat 5: 234-248, 2002.

4. Franke TF, Hornik CP, Segev L, Shostak GA and Sugimoto C: PI3K/Akt and apoptosis: size matters. Oncogene 22: 8983-8998, 2003.

5. Xu Q, Simpson SE, Scialla TJ, Bagg A and Carroll M: Survival of acute myeloid leukemia cells requires PI3 kinase activation. Blood 102: 972-980, 2003.

6. Tamburini J, Chapuis N, Bardet V, et al: Mammalian target of rapamycin (mTOR) inhibition activates phosphatidylinositol 3-kinase/Akt by up-regulating insulin-like growth factor-1 receptor signaling in acute myeloid leukemia: rationale for therapeutic inhibition of both pathways. Blood 111: 379-382, 2008.

7. Zeng Z, Shi YX, Tsao T, et al: Targeting of $\mathrm{mTORC} 1 / 2$ by the mTOR kinase inhibitor PP242 induces apoptosis in AML cells under conditions mimicking the bone marrow microenvironment. Blood 120: 2679-2689, 2012.

8. Shrimali D, Shanmugam MK, Kumar AP, et al: Targeted abrogation of diverse signal transduction cascades by emodin for the treatment of inflammatory disorders and cancer. Cancer Lett 341: 139-149, 2013.

9. Wu L, Cai B, Zheng S, Liu X, Cai H and Li H: Effect of emodin on endoplasmic reticulum stress in rats with severe acute pancreatitis. Inflammation 36: 1020-1029, 2013.

10. Tamokou Jde D, Chouna JR, Fischer-Fodor E, et al: Anticancer and antimicrobial activities of some antioxidant-rich Cameroonian medicinal plants. PloS One 8: e55880, 2013.

11. Zhang L, Lau YK, Xia W, Hortobagyi GN and Hung MC: Tyrosine kinase inhibitor emodin suppresses growth of HER-2/ neu-overexpressing breast cancer cells in athymic mice and sensitizes these cells to the inhibitory effect of paclitaxel. Clin Cancer Res 5: 343-353, 1999.

12. Brown M, Bellon M and Nicot C: Emodin and DHA potently increase arsenic trioxide interferon-alpha-induced cell death of HTLV-I-transformed cells by generation of reactive oxygen species and inhibition of Akt and AP-1. Blood 109: 1653-1659, 2007.

13. Wei WT, Chen H, Ni ZL, et al: Antitumor and apoptosispromoting properties of emodin, an anthraquinone derivative from Rheum officinale Baill, against pancreatic cancer in mice via inhibition of Akt activation. Int J Oncol 39: 1381-1390, 2011. 
14. Huang LY, Hu JD, Chen XJ, Zhu LF and Hu HL: Effects of emodin on the proliferation inhibition and apoptosis induction in HL-60 cells and the involvement of c-myc gene. Zhonghua Xue Ye Xue Za Zhi 26: 348-351, 2005.

15. Chen YY, Zheng HY, Hu JD, et al: Inhibitory effects of emodin on drug-resistant HL-60/ADR cell proliferation and its induction of apoptosis. Zhongguo Shi Yan Xue Ye Xue Za Zhi 15: 955-960, 2007.

16. Chen YY, Li J, Hu JD, et al: Reversing effects of emodin on multidrug resistance in resistant HL-60/ADR cells. Zhongguo Shi Yan Xue Ye Xue Za Zhi 21: 1413-1422, 2013.

17. Lin M, Hu J, Liu T, Li J, Chen B and Chen X: Knockdown of nucleophosmin by RNA interference reverses multidrug resistance in resistant leukemic HL-60 cells. Immunobiology 218 $1147-1154,2013$

18. Hu J, Lin M, Liu T, Li J, Chen B and Chen Y: DIGE-based proteomic analysis identifies nucleophosmin/B23 and nucleolin C23 as over-expressed proteins in relapsed/refractory acute leukemia. Leuk Res 35: 1087-1092, 2011.

19. Tamburini J,Elie C, Bardet V, et al: Constitutive phosphoinositide 3-kinase/Akt activation represents a favorable prognostic factor in de novo acute myelogenous leukemia patients. Blood 110 1025-1028, 2007.

20. Martelli AM, Evangelisti C, Chiarini F and McCubrey JA: The phosphatidylinositol 3-kinase/Akt/mTOR signaling network as a therapeutic target in acute myelogenous leukemia patients. Oncotarget 1: 89-103, 2010.

21. Grandage VL, Gale RE, Linch DC and Khwaja A: PI3-kinase/ Akt is constitutively active in primary acute myeloid leukaemia cells and regulates survival and chemoresistance via NF-kappaB MAPkinase and p53 pathways. Leukemia 19: 586-594, 2005.

22. Niu C, Yan H, Yu T, et al: Studies on treatment of acute promyelocytic leukemia with arsenic trioxide: remission induction, follow-up, and molecular monitoring in 11 newly diagnosed and 47 relapsed acute promyelocytic leukemia patients. Blood 94 3315-3324, 1999.

23. Mathews V, George B, Chendamarai E, et al: Single-agent arsenic trioxide in the treatment of newly diagnosed acute promyelocytic leukemia: long-term follow-up data. J Clin Oncol 28: 3866-3871, 2010.

24. Ghavamzadeh A, Alimoghaddam K, Rostami S, et al: Phase II study of single-agent arsenic trioxide for the front-line therapy of acute promyelocytic leukemia. J Clin Oncol 29: 2753-2757, 2011.

25. Ma YN, Chen MT, Wu ZK, et al: Emodin can induce K562 cells to erythroid differentiation and improve the expression of globin genes. Mol Cell Biochem 382: 127-136, 2013.
26. Muto A, Hori M, Sasaki Y, et al: Emodin has a cytotoxic activity against human multiple myeloma as a Janus-activated kinase 2 inhibitor. Mol Cancer Ther 6: 987-994, 2007.

27. Yan Y, Su X, Liang Y, et al: Emodin azide methyl anthraquinone derivative triggers mitochondrial-dependent cell apoptosis involving in caspase-8-mediated Bid cleavage. Mol Cancer Ther 7: 1688-1697, 2008.

28. Tauchi T, Sumi M, Nakajima A, Sashida G, Shimamoto T and Ohyashiki K: BCL-2 antisense oligonucleotide genasense is active against imatinib-resistant BCR-ABL-positive cells. Clin Cancer Res 9: 4267-4273, 2003.

29. Kim R, Emi M, Tanabe K and Toge T: Preclinical evaluation of antisense bcl-2 as a chemosensitizer for patients with gastric carcinoma. Cancer 101: 2177-2186, 2004.

30. Karnak D and Xu L: Chemosensitization of prostate cancer by modulating Bcl-2 family proteins. Current Drug Targets 11: 699-707, 2010.

31. Parrondo R, de Las Pozas A, Reiner T and Perez-Stable C: ABT-737, a small molecule Bcl-2/Bcl-xL antagonist, increases antimitotic-mediated apoptosis in human prostate cancer cells. PeerJ 1: e144, 2013.

32. Nasr R, Guillemin MC, Ferhi O, et al: Eradication of acute promyelocytic leukemia-initiating cells through PML-RARA degradation. Nat Med 14: 1333-1342, 2008.

33. Hu J, Liu YF, Wu CF, et al: Long-term efficacy and safety of all-trans retinoic acid/arsenic trioxide-based therapy in newly diagnosed acute promyelocytic leukemia. Proc Natl Acad Sci USA 106: 3342-3347, 2009.

34. Isakson P, Bjoras M, Boe SO and Simonsen A: Autophagy contributes to therapy-induced degradation of the PML/RARA oncoprotein. Blood 116: 2324-2331, 2010.

35. Zhang XW, Yan XJ, Zhou ZR, et al: Arsenic trioxide controls the fate of the PML-RARalpha oncoprotein by directly binding PML. Science 328: 240-243, 2010.

36. Harris MN, Ozpolat $\mathrm{B}$, Abdi F, et al: Comparative proteomic analysis of all-trans-retinoic acid treatment reveals systematic posttranscriptional control mechanisms in acute promyelocytic leukemia. Blood 104: 1314-1323, 2004.

37. Bayascas JR and Alessi DR: Regulation of Akt/PKB Ser473 phosphorylation. Mol Cell 18: 143-145, 2005.

38. Sarbassov DD, Guertin DA, Ali SM and Sabatini DM: Phosphorylation and regulation of Akt/PKB by the rictor-mTOR complex. Science 307: 1098-1101, 2005. 